\title{
On topological field theory representation of higher analogs of classical special functions *
}

\author{
Anton A. Gerasimov and Dimitri R. Lebedev
}

\begin{abstract}
Looking for a quantum field theory model of Archimedean algebraic geometry a class of infinite-dimensional integral representations of classical special functions was introduced. Precisely the special functions such as Whittaker functions and $\Gamma$-function were identified with correlation functions in topological field theories on a two-dimensional disk. Mirror symmetry of the underlying topological field theory leads to a dual finite-dimensional integral representations reproducing classical integral representations for the corresponding special functions. The mirror symmetry interchanging infinite- and finite-dimensional integral representations provides an incarnation of the local Archimedean Langlands duality on the level of classical special functions.

In this note we provide some directions to higher-dimensional generalizations of our previous results. In the first part we consider topological field theory representations of multiple local $L$-factors introduced by Kurokawa and expressed through multiple Barnes's $\Gamma$-functions. In the second part we are dealing with generalizations based on consideration of topological Yang-Mills theories on non-compact four-dimensional manifolds. Presumably, in analogy with the mirror duality in two-dimensions, $S$-dual description should be instrumental for deriving integral representations for a particular class of quantum field theory correlation functions and thus providing a new interesting class of special functions supplied with canonical integral representations.
\end{abstract}

\section{Introduction}

In GLO2, GLO3, GLO4, GLO5, G] a topological field theory framework for a description of the Archimedean algebraic geometry was proposed. As a first step [GLO2, GLO3 local Archimedean $L$-factors were interpreted as correlation functions in two-dimensional equivariant topological field theories on a disk. It was demonstrated that the local Archimedean Langlands correspondence between various constructions of $L$-factors (see e.g. [B], L]) is realized as mirror symmetry on the level of underlying topological field theories GLO3]. These results were generalized to a class of Whittaker functions in [GLO4]. Presumably this picture holds in full generality and provides a realization of the Archimedean Langlands duality for a generic Whittaker function. Moreover, one can expect that the approach of [GLO2, GLO3], GLO4], GLO5], G] will lead to an interpretation of all basic constructions of Archimedean algebraic geometry in terms of twodimensional topological field theories thus providing a clue to a natural formulation of the geometry over Archimedean fields.

*Extended version of a talk given by the first author at Quantum field theory and representation theory, October, 2010, Moscow, Russia. 
Given the proposed connection of the Archimedean geometry with two-dimensional topological field theories it is natural to ask what is a special role of two dimensions in these considerations and are there any signs of possible generalizations of [GLO2], GLO3], GLO4] to other dimensions. The case of zero dimension was considered in GL1]. This note is a very preliminary discussion of a large project of higher dimensional generalizations of [GLO2], GLO3], GLO4]. In the first part we propose a description of higher analogs of local Archimedean $L$-factors introduced by Kurokawa Ku1, $\mathrm{Ku} 2$ (see also $\mathrm{Ma}$ ) in terms of equivariant topological field theories with quadratic actions. The Kurokawa $L$-factors shall be considered as basic building blocks of higher-dimensional generalizations of the Mellin-Barnes representations $\mathrm{KL}$. We also speculate on a dual description generalizing type $B$ description of the standard local Archimedean $L$-factors GLO3]. Eventually this should lead to a higher-dimensional generalization of the local Archimedean Langlands correspondence.

In the second part of this note we pursue another (but related) direction of generalization of GLO4 relying on the four-dimensional topological field theories obtained by twisting of $\mathcal{N}=2$ SUSY Yang-Mills theory on non-compact four-dimensional manifolds. These theories have $S$ dual descriptions in terms of theories of abelain gauge fields interacting with monopoles. There exists a class of correlation functions in twisted $\mathcal{N}=2$ SUSY Yang-Mills theories on non-compact four-dimensional manifolds computable by direct counting gauge theory instantons [LNS], [N], [NO], NY]. This class of correlation functions shall be considered as a close analog of the class of correlation functions in type $A$ topological sigma models considered in [GLO4]. Thus this class of correlation functions in four-dimensional topological field theories should also have natural integral representations similar to those derived in GLO4 for two-dimensional topological sigma models. In two-dimensional case the integral representations of correlation functions in topological sigma models provide a direct relation with the mirror dual formulations in terms of topological Landau-Ginzburg theories. One expects that in four-dimensions the integral representations of the instanton counting functions provide direct links with the dual monopole descriptions of the gauge theories (captured effectively by the Seiberg-Witten prepotential). This should also provide integral representations of new special functions generalizing Whittaker functions (see [BE] for related considerations). In this note we discuss what can be considered as a proper set-up for a verification of these hopes. We consider only the case of non-compact four-manifolds with an action of $S^{1}$ isometry leaving more general cases e.g. allowing actions of $S^{1} \times S^{1}$ for future publication GL2]. Meanwhile, as a simple exercise, we explicitly construct an integral representation of the Mellin-Barnes type for a limit of equivariant instanton counting function (first introduced in [LNS]) describing vortex in two-dimensional models (see e.g. [Shad]).

Let us make a short comment on (a small part of) modern literature on topological / supersymmetric quantum field theories relevant to the subject of this note. One of the key points of the constructions of [GLO2, GLO3, GLO4 was the use the equivariant setting with respect to a group of global symmetries including space-time rotations. Although a relation between $S^{1}$ equivariance and coupling of two-dimensional topological sigma models with topological gravity was well-known, the effective use of the $S^{1}$-equivaraint extensions of topological sigma model was initiated by Kontsevich [K] and further advanced in [Gi1], Gi2], Gi3]. The four-dimensional analog of this approach was considered in [LNS] in the context of instanton counting on $\mathbb{R}^{4}$ and was convincingly related in $[\mathrm{N}],[\mathrm{NO}],[\mathrm{NY}]$ to the Seiberg-Witten solution [SW] of $\mathcal{N}=2$ SUSY YangMills theory. An identification of a correlation function on a disk in a class of two-dimensional topological field theories with solutions of quantum integrable systems was proposed in GS1 using previous findings in MNS] and was argued in GS2 to be a general phenomena. Many examples, including four-dimensional cases relevant to considerations of this note were considered in [NS] (see 
also [NW]). For a detailed discussion of supersymmetric/ topological quantum field theories on non-compact manifolds see [GW]. In the remarkable paper [AGT] a relation between correlation functions of four-dimensional SUSY gauge theories and correlation functions in two-dimensional models was proposed. This in particular implies that the class of special functions we are looking for shall include building blocks of correlation functions in two-dimensional theories (such as conformal Toda theories). Note also that counting of BPS states in SUSY quantum field theories [R1], KMMR], R2] leads to generalized Mellin-Barnes type integral representations in terms of combinations of double $\Gamma$-functions known as elliptic sin functions [DO] (see also [S] for various relevant identities). This provides another example of special functions of a new kind related with quantum field theories. Finally, in a recent paper [W3] a particular class of correlation functions in topological gauge field theories on non-compact four-manifolds was proposed partially overlapping with the discussions in the second part of this note.

Acknowledgments: The research was supported by Grant RFBR-09-01-93108-NCNIL-a. The research of AG was also partly supported by Science Foundation Ireland grant.

\section{Kurokawa multiple $L$-factors via topological field theory}

In GLO2 a representation of local Archimedean $L$-factors as correlation functions in equivariant topological sigma models on a disk with target spaces $\mathbb{C}^{\ell+1}$ was constructed. The local Archimedean $L$-factors are basically given by products of $\Gamma$-functions and [GLO2] uses a realization of the $\Gamma$ function as an inverse of a regularized infinite-dimensional determinant obtained by taking an infinite-dimensional Gaussian integral. This representation can be straightforwardly generalized to higher dimensions. Multiple $\Gamma$-functions introduced by Barnes $[\mathrm{Ba}$ ] allow a representation as inverse regularized infinite-dimensional determinants. Below we recast this representation into a framework of higher-dimensional topological equivariant field theories. Kurokawa proposed to use multiple $\Gamma$-functions for construction of higher analogs of $L$-factors and rise the question of their arithmetic interpretation [Ku1], $\mathrm{Ku} 2$ ] (see also [Ma]). Thus the topological field theory representation of multiple $\Gamma$-functions provides a topological field theory representation of higher local $L$-factors generalizing [GLO2] to higher dimensions.

We expect that the mirror dual representation of [GLO3] has also a generalization to higher dimensions. This provides an instance of a higher dimensional analog of the local Archimedean Langlands correspondence. In this Section we only briefly touch this topic by calculating the multiple $\Gamma$-function via fixed point localization of the corresponding topological field theory integral (as it was demonstrated in GLO3 this directly leads to mirror dual description for classical $\Gamma$ functions).

\section{$1.1 \quad$ Multiple Gamma-functions}

Let us first recall the basic constructions of the hierarchy of $\Gamma$-functions $[\mathrm{Ba}$. The simplest $\Gamma$ function (called elementary $\Gamma$-function in [GL1]) is given by

$$
\Gamma_{0}(s)=\frac{1}{s} \text {. }
$$

The standard $\Gamma$-function can be considered as a next element of the hierarchy of $\Gamma$-functions. Actually it is more natural to introduce a slightly modified $\Gamma$-function

$$
\Gamma_{1}(s \mid \omega)=(2 \pi)^{-\frac{1}{2}} \omega^{\frac{s}{\omega}-\frac{1}{2}} \Gamma(s / \omega), \quad \omega>0,
$$


satisfying the functional equation

$$
\frac{\Gamma_{1}(s \mid \omega)}{\Gamma_{1}(s+\omega \mid \omega)}=\Gamma_{0}(s)
$$

The modified $\Gamma$-function can be also expressed through a regularized infinite product

$$
\Gamma_{1}(s \mid \omega)=\left[\prod_{n \in \mathbb{Z}_{\geq 0}}(s+n \omega)^{-1}\right]_{r e g}:=\exp \left(\left.\frac{\partial}{\partial \nu} \zeta(s, \nu \mid \omega)\right|_{\nu=0},\right.
$$

where $\zeta(s, \nu \mid \omega)$ is defined as an analytic continuation of the infinite sum

$$
\zeta(s, \nu \mid \omega)=\sum_{n \in \mathbb{Z}_{\geq}} \frac{1}{(s+n \omega)^{\nu}}, \quad \operatorname{Re}(\nu)>1 .
$$

Another useful way to define (modified) $\Gamma$-function is via the Gauss integral representation of its logarithm

$$
\ln \Gamma_{1}(s \mid \omega)=-\gamma\left(\frac{s}{\omega}-\frac{1}{2}\right)+\int_{\mathcal{C}} \frac{d t}{2 \pi \imath t} \frac{e^{-s t} \ln (-t)}{\left(1-e^{-\omega t}\right)}
$$

where $\gamma$ is the Euler constant and $\mathcal{C}$ is the Hankel contour starting at $+\infty$ enclosing $t=0$ counterclockwise and returning to $+\infty$.

The elementary $\Gamma$-function (1.1) can be obtained as a limit of the modified $\Gamma$-function

$$
\Gamma_{0}(s)=\lim _{\omega \rightarrow \infty}\left(\frac{\omega}{2 \pi}\right)^{-\frac{1}{2}} \Gamma_{1}(s \mid \omega)
$$

taking into account (1.3) and $\Gamma_{1}(\omega \mid \omega)=(\omega / 2 \pi)^{1 / 2}$. Let $\underline{\omega}=\left(\omega_{1}, \ldots, \omega_{r}\right)$ be an array of positive real numbers. The multiple $\Gamma$-functions are defined as infinite products

$$
\Gamma_{r}(s \mid \underline{\omega})=\left[\prod_{\underline{n} \in \mathbb{Z}_{\geq 0}^{r}}(s+\langle\underline{n}, \underline{\omega}\rangle)^{-1}\right]_{r e g}=\left.\frac{\partial}{\partial \nu} \zeta_{r}(s, \nu \mid \underline{\omega})\right|_{\nu=0}, \quad \underline{\omega}=\left(\omega_{1}, \ldots, \omega_{r}\right), \quad \underline{n}=\left(n_{1}, \ldots, n_{r}\right),
$$

regularized using analytic continuation of the $\zeta$-function $[\mathrm{Ba}]$

$$
\zeta_{r}(s, \nu \mid \underline{\omega})=\sum_{\underline{n} \in \mathbb{Z}_{\geq}^{r}} \frac{1}{(s+\langle\underline{n}, \underline{\omega}\rangle)^{\nu}}, \quad \operatorname{Re}(\nu)>r .
$$

Thus defined multiple $\Gamma$-functions satisfy the following defining equations:

$$
\frac{\Gamma_{r}(s \mid \underline{\omega})}{\Gamma_{r}\left(s+\omega_{i} \mid \underline{\omega}\right)}=\Gamma_{r-1}\left(s \mid \underline{\omega}-\left\{\omega_{i}\right\}\right), \quad i=1, \ldots, r
$$

and more generally

$$
\prod_{\underline{\epsilon}} \Gamma_{r}(s+\langle\underline{\omega}, \underline{\epsilon}\rangle \mid \underline{\omega})^{(-1)^{|\underline{\epsilon}|}}=s^{-1}
$$

where $\underline{\epsilon}=\left(\epsilon_{1}, \ldots, \epsilon_{r}\right), \epsilon_{i}=0,1$ and $|\underline{\epsilon}|=\sum_{i} \epsilon_{i}$. 
Multiple $\Gamma$-functions allow integral representation generalizing the classical Gauss integral representation (1.6)

$$
\ln \Gamma_{r}(s \mid \underline{\omega})=\frac{(-1)^{r}}{r !} \gamma B_{r, r}(s)+\int_{\mathcal{C}} \frac{d t}{2 \pi \imath t} \frac{e^{-s t} \ln (-t)}{\prod_{a=1}^{r}\left(1-e^{-\omega_{a} t}\right)},
$$

where $\mathcal{C}$ is the Hankel contour. Here $\gamma$ is the Euler constant and the Bernoulli polynomial $B_{r, k}(s)$ are defined by the following generating series:

$$
\frac{t^{r} e^{z t}}{\prod_{k=1}^{r}\left(e^{\omega_{k} t}-1\right)}=\sum_{k=0}^{+\infty} B_{r, k}(z, \underline{\omega}) \frac{t^{k}}{k !} .
$$

The following limiting relation between multiple $\Gamma$-functions holds:

$$
\Gamma_{r-1}\left(s \mid \underline{\omega}-\left\{\omega_{r}\right\}\right)=\lim _{\omega_{r} \rightarrow \infty} \frac{\Gamma_{r}(s \mid \underline{\omega})}{\Gamma_{r}\left(\omega_{r} \mid \underline{\omega}\right)} .
$$

Let us note that hierarchy of multiple $\Gamma$-functions allows a natural $q$-deformation. The $q$-deformed multiple $\Gamma$-function is defined as an infinite product

$$
\Gamma_{r}(t \mid \underline{q})=\prod_{\underline{n} \in \mathbb{Z}_{\geq 0}^{r}} \frac{1}{\left(1-t q_{1}^{n_{1}} \cdots q_{r}^{n_{r}}\right)},
$$

where $\underline{q}=\left(q_{1}, \ldots, q_{r}\right)$ and we imply $\left|q_{j}\right|<1, j=1, \ldots, r$. Under these conditions the product absolutely converges and no regularization is needed. The $q$-deformed multiple $\Gamma$-functions can be also represented as an infinite determinant

$$
\Gamma_{r}(t \mid \underline{q})=\operatorname{det}_{\mathbb{C}\left[z_{1}, \ldots, z_{r}\right]} \frac{1}{\left(1-t q_{1}^{d_{1}} \cdots q_{r}^{d_{r}}\right)},
$$

where the mutually commuting operators $d_{j}$ act as follows:

$$
d_{j} \cdot z_{1}^{n_{1}} \cdots z_{r}^{n_{r}}=n_{j} \cdot z_{1}^{n_{1}} \cdots z_{r}^{n_{r}} .
$$

One can represent $q$-deformed multiple $\Gamma$-functions as traces using the standard algebraic identities. Let $\mathcal{A}_{r}$ be the space of polynomials of the variable $Z_{i, n}, i=1, \ldots, r, n \in \mathbb{Z}_{\geq}$. Then we have the following representation:

$$
\Gamma_{r}(t \mid \underline{q})=\operatorname{Tr}_{\mathcal{A}_{r}} t^{D} q_{1}^{d_{1}} \cdots q_{r}^{d_{r}}
$$

where the actions of the mutually commuting operators $D$ and $d_{1}, \ldots, d_{r}$ on $\mathcal{A}_{r}$ are induced by the following actions on the generators of the polynomial ring:

$$
d_{j} \cdot Z_{i, n}=\delta_{i j} n Z_{i n}, \quad D \cdot Z_{j n}=Z_{j n} .
$$

The multiple $q$-deformed $\Gamma$-functions satisfy $q$-analogs of the functional relations (1.10)

$$
\prod_{\underline{\epsilon}} \Gamma_{r}\left(t \prod_{i=1}^{r} q_{i}^{\epsilon_{i}} \mid \underline{q}\right)^{(-1)|\epsilon|}=\frac{1}{1-t} .
$$

These functional relations (1.19) allow a simple interpretation in terms of coherent sheaves on $\mathbb{C}^{r}$. Consider for example the following exact sequence of $\mathbb{C}[z]$-modules:

$$
0 \longrightarrow z \mathbb{C}[z] \longrightarrow \mathbb{C}[z] \longrightarrow \mathbb{C} \longrightarrow 0
$$


Using multiplicative property of determinants with respect to exact sequences we have

$$
\operatorname{det}_{\mathbb{C}}(1-t) \operatorname{det}_{z \mathbb{C}[z]}\left(1-t q^{d}\right)=\operatorname{det}_{\mathbb{C}[z]}\left(1-t q^{d}\right),
$$

where $d \cdot z^{n}=n z^{n}$. Taking into account the definition (1.14) of $q$-deformed $\Gamma$-functions we have the functional relation

$$
\frac{\Gamma_{1}(t \mid q)}{\Gamma_{1}(q t \mid q)}=\Gamma_{0}(t)
$$

where

$$
\Gamma_{0}(t)=\frac{1}{1-t}, \quad \Gamma_{1}(t \mid q)=\frac{1}{\prod_{n=0}^{\infty}\left(1-t q^{n}\right)}
$$

For $r=2$ consider the Koszul resolution of the skyscraper sheaf $\mathcal{O}_{z=0}$ at $0 \in \mathbb{C}^{2}$

$$
0 \longrightarrow \mathbb{C}\left[z_{1}, z_{2}\right] \longrightarrow z_{1} \mathbb{C}\left[z_{1}, z_{2}\right] \oplus z_{2} \mathbb{C}\left[z_{1}, z_{2}\right] \longrightarrow \mathbb{C}\left[z_{1}, z_{2}\right] \longrightarrow \mathbb{C} \longrightarrow 0 .
$$

Let $d_{1}, d_{2}$ be commuting operators acting on polynomials via $d_{i} \cdot z_{j}^{n_{j}}=\delta_{i j} n_{j} z_{j}^{n_{j}}$. Taking determinant of the operator $\left(1-t q_{1}^{d_{1}} q_{2}^{d_{2}}\right)$ acting on various terms of the exact sequence we arrive at the following functional relation:

$$
\frac{\Gamma_{2}\left(t q_{1} q_{2} \mid q_{1}, q_{2}\right) \Gamma_{2}\left(t \mid q_{1}, q_{2}\right)}{\Gamma_{2}\left(t q_{1} \mid q_{1}, q_{2}\right) \Gamma_{2}\left(t q_{2} \mid q_{1}, q_{2}\right)}=\frac{1}{1-t}
$$

which is a particular instance of (1.19).

\subsection{Topological field theory interpretation}

Now we provide a simple interpretation of the multiple $\Gamma$-functions (and thus Kurokawa generalized local Archimedean $L$-factors) as correlation functions in equivariant topological field theories thus generalizing the results of GLO2 to higher dimensions. We start with a representation of multiple $\Gamma$-functions as properly regularized symplectic volumes of infinite-dimensional spaces. Consider the space $\mathcal{M}\left(D^{r}, \mathbb{C}^{\ell+1}\right)$ of holomorphic maps of the polydisk $D_{r}=\left\{\left(z_{1}, \ldots, z_{r}\right) \in \mathbb{C}^{r}|| z_{a} \mid \leq 1\right\}$ into the vector space $\mathbb{C}^{\ell+1}$. There are natural actions of $U_{\ell+1}$ and real $r$-dimensional torus $T_{r}=S^{1} \times \cdots \times S^{1}$ on $\mathcal{M}\left(D^{r}, \mathbb{C}^{\ell+1}\right)$. The action of $U_{\ell+1}$ is induced from the standard action on $\mathbb{C}^{\ell+1}$ and $T_{r}$ acts by rotation of $D_{r}$

$$
z_{a} \rightarrow e^{\imath \alpha_{a}} z_{a}, \quad a=1, \ldots, r .
$$

The space of maps has natural symplectic structure

$$
\Omega=\frac{\imath}{2(2 \pi)^{r}} \int_{\left|z_{a}\right|=1} \prod_{a=1}^{r} d \sigma_{a} \sum_{j=1}^{\ell+1} \delta \varphi^{j}(z) \wedge \delta \bar{\varphi}^{j}(z), \quad z_{a}=r_{a} e^{\imath \sigma_{a}}
$$

induced by the standard symplectic structure on $\mathbb{C}^{\ell+1}$

$$
\omega=\frac{\imath}{2} \sum_{j=1}^{r} d z_{j} \wedge d \bar{z}_{j}
$$


The action of $U_{\ell+1} \times T_{r}$ is Hamiltonian with respect to (1.26) and the corresponding momenta for the diagonal subgroup $U_{1}^{\ell+1} \subset U_{\ell+1}$ and $T_{r}$ are given by

$$
\begin{aligned}
& H_{b}=\frac{\imath}{2(2 \pi)^{r}} \int_{\left|z_{a}\right|=1} \prod_{a=1}^{r} d \sigma_{a} \sum_{j=1}^{\ell+1} \bar{\varphi}^{j} \partial_{\sigma_{b}} \varphi^{j}, \quad b=1, \ldots, r, \\
& H_{j}=-\frac{1}{2(2 \pi)^{r}} \int_{\left|z_{a}\right|=1} \prod_{a=1}^{r} d \sigma_{a}\left|\varphi^{j}\right|^{2}, \quad j=1, \ldots,(\ell+1) .
\end{aligned}
$$

We define $T_{r} \times U_{\ell+1}$-equivariant volume of $\mathcal{M}\left(D_{r}, \mathbb{C}^{\ell+1}\right)$ as the following infinite-dimensional integral:

$$
Z(\underline{\lambda}, \underline{\omega})=\int_{\mathcal{M}\left(D_{r}, \mathbb{C}^{\ell+1}\right)} e^{\Omega-\sum_{j=1}^{\ell+1} \lambda_{j} H_{j}-\sum_{a=1}^{r} \omega_{a} H_{a}}, \quad \omega_{a}>0
$$

where $\underline{\lambda}=\left(\lambda_{1}, \ldots, \lambda_{\ell+1}\right), \underline{\omega}=\left(\omega_{1}, \ldots, \omega_{\ell+1}\right)$ and the infinite-dimensional Gaussian integrals are understood via $\zeta$-function regularization. Straightforward calculations similar to the one in GLO2] give

$$
Z(\underline{\lambda}, \underline{\omega})=\prod_{j=1}^{\ell+1} \Gamma_{r}\left(\lambda_{j} \mid \underline{\omega}\right) .
$$

It is easy to write down representation for the equivariant symplectic volume (1.30) using formalism of topological field theories. Consider a quantum field theory on $D_{r}$ with the following set of fields:

$$
\left(\varphi^{j}, \bar{\varphi}^{j}, \chi^{j}, \bar{\chi}^{j}, \psi^{j a}, \bar{\psi}^{j a}, F^{j a}, \bar{F}^{j a}\right), \quad j=1, \ldots(\ell+1), \quad a=1, \ldots r,
$$

where $\varphi$ and $F$ are even and $\psi$ and $\chi$ are odd fields. Note that the complex functions $(\varphi, F, \psi, \chi)$ can be written in terms of real functions

$$
\left(\varphi^{A}, \chi^{A}, \psi^{A \mu}, F^{A \mu}\right), \quad A=1, \ldots 2(\ell+1), \quad \mu=1, \ldots 2 r,
$$

with additional constraints

$$
\psi_{\mu}^{A}+\left(J_{2}\right)_{\mu}^{\nu} \psi_{\nu}^{B}\left(J_{1}\right)_{B}^{A}=0, \quad F_{\mu}^{A}+\left(J_{2}\right)_{\mu}^{\nu} F_{\nu}^{B}\left(J_{1}\right)_{B}^{A}=0 .
$$

Here $J_{1} \in \operatorname{End}\left(\mathbb{R}^{2 r}\right)$ and $J_{2} \in \operatorname{End}\left(\mathbb{R}^{2 \ell+2}\right)$ are standard complex structures on $\mathbb{C}^{r}$ and $\mathbb{C}^{\ell+1}$ correspondingly.

Let us pick a $T_{r}$-invariant metric $h$ on $D_{r}$. Consider a topological theory with the following action functional:

$$
S_{0}=\delta_{0}\left(\int_{D^{r}} d^{2 r} z \sqrt{h} \mathcal{V}\right)=\int_{D^{r}} d^{2 r} z \sqrt{h} h^{\mu \nu}\left(F_{\mu}^{A} \partial_{\nu} \phi^{A}+\psi_{\mu}^{A} \partial_{\nu} \chi^{A}\right)
$$

where

$$
\mathcal{V}=h^{\mu \nu} \psi_{\mu}^{A} \partial_{\nu} \phi^{A}
$$

and $\delta_{0}$ is the BRST operator defined by the relations

$$
\delta_{0} \phi^{A}=\chi^{A}, \quad \delta_{0} \chi^{A}=0, \quad \delta_{0} \psi_{\mu}^{A}=F_{\mu}^{A}, \quad \delta_{0} F_{\mu}^{A}=0 .
$$


The operator $\delta_{0}$ is obviously nilpotent $\delta_{0}^{2}=0$.

We are interested in construction of $U_{\ell+1} \times T_{r}$-equivariant generalization of the theory with the action (1.35). Let us start with $T_{r}$-equivariance. The $T_{r}$-equivariant differential is given by standard equivariant extension of the de Rham differential

$$
\delta_{T_{r}}=\delta_{0}+\sum_{a=1}^{r} \omega_{a} \iota_{v_{a}}, \quad v_{a}=\imath\left(z_{a} \frac{\partial}{\partial z_{a}}-\bar{z}_{a} \frac{\partial}{\partial \bar{z}_{a}}\right),
$$

and $\left(\omega_{1}, \ldots, \omega_{r}\right)$ is an element of the Lie algebra $\operatorname{Lie}\left(T_{r}\right)$. Consider the action of the diagonal subgroup $U(1)^{\ell+1}$ on $\mathbb{C}^{\ell+1}$ given by

$$
e^{\imath \alpha_{j}}: \varphi^{k} \longrightarrow e^{\imath \alpha_{j} \delta_{j, k}} \varphi^{k}, \quad j, k=1, \ldots,(\ell+1) .
$$

Now $U_{1}^{\ell+1} \times T_{r}$-equivariant generalization of the BRST operator (1.37) is given by

$$
\begin{gathered}
\delta \varphi^{j}=\chi^{j}, \quad \delta \chi^{j}=-\left(\sum_{a=1}^{r} \omega_{a} i_{v_{a}} d \varphi^{j}+\imath \lambda_{j} \varphi^{j}\right), \\
\delta \psi^{j}=F^{j}, \quad \delta F^{j}=-\left(\sum_{a=1}^{r} \omega_{a} \mathcal{L}_{v_{a}} \psi^{j}+\imath \lambda_{j} \psi^{j}\right) .
\end{gathered}
$$

where $\left(\lambda_{1}, \ldots, \lambda_{\ell+1}\right)$ is an element of Lie algebra $\operatorname{Lie}\left(U_{1}^{\ell+1}\right)$. Consider the following $U_{1}^{\ell+1} \times T_{r^{-}}$ invariant $\delta$-closed form on the space of maps $D_{r} \rightarrow \mathbb{C}^{\ell+1}$ :

$$
\mathcal{O}=\frac{\imath}{2(2 \pi)^{r}} \int_{\left|z_{a}\right|=1} \prod_{a=1}^{r} d \sigma_{a}\left(\sum_{j=1}^{\ell+1} \chi^{j} \bar{\chi}^{j}+\imath \lambda_{j}\left|\varphi^{j}\right|^{2}+\sum_{a=1}^{r} \omega_{a} \bar{\varphi}^{j} \partial_{\sigma_{a}} \varphi^{j}\right) .
$$

Now we would like to calculate the functional integral with the following modified action

$$
S=\int_{D^{r}} d^{2 r} z \sqrt{h} h^{\mu \nu}\left(F_{\mu}^{A} \partial_{\nu} \phi^{A}+\psi_{\mu}^{A} \partial_{\nu} \chi^{A}\right)+\mathcal{O} .
$$

Integrating over $F$ we restrict the fields $\phi$ to the subspace

$$
\bar{\partial} \phi^{i}=0, \quad \partial \bar{\phi}^{i}=0,
$$

and similarly for integration over $\psi$. Further integral over holomorphic fields $\phi$ and $\chi$ reduces to the equivariant volume integral (1.30). Thus the functional integral with the action (1.42) gives the integral representation for multiple $\Gamma$-function

$$
Z(\underline{\lambda} \mid \underline{\omega})=\prod_{j=1}^{\ell+1} \Gamma_{r}\left(\lambda_{j} \mid \underline{\omega}\right)
$$

Let us also note that the topological field theory with the action (1.42) for $r=2$ and $\ell+1=2$ can be interpreted as an $U_{2} \times T_{2}$-equivariant extension of the theory of $\mathcal{N}=2$ SUSY hypermultiplet.

Recall that classical $\Gamma$-function provides a basic building block of the Mellin-Barnes integral representation of various special functions $[\mathrm{KL}$, GKL]. Given a quantum field theory representation of multiple $\Gamma$-functions, one might expect that the special functions allowing the Mellin-Barnes integral representations have natural higher-dimensional generalizations expressed in terms of multiple $\Gamma$-functions and related with correlation functions in higher-dimensional equivariant topological field theories. 


\subsection{Multiple Euler integrals via fixed point calculation}

In the case of two-dimensional disk (i.e. for $r=1$ ) the functional integral in the left hand side of (1.44) was interpreted as a correlation function in type $A$ topological sigma model i.e. as a topological sigma model obtained by type $A$ twisting from a $\mathcal{N}=2$ SUSY sigma model [GLO1]. One can consider a mirror dual type $B$ topological sigma model of the Landau-Ginzburg type. This dual description naturally leads to the Euler integral representation of the classical $\Gamma$-function. In [GLO3] it was demonstrated that the type $B$ dual description can be obtained directly from the type $A$ functional integral using fixed point localization technique. Below we provide a heuristic derivation of the multiple analog of the Euler integral representation of multiple $\Gamma$-functions applying fixed point localization to the infinite-dimensional integral (1.30). Thus obtained integral representations of multiple $\Gamma$-functions shall naturally arise in mirror dual description of the topological field theories with the actions (1.42).

Let us first briefly recall the derivation of the Euler integral representation of the classical $\Gamma$-function via fixed point localization [GLO3]. Precisely we are going to derive an expression for the Fourier transform of $\Gamma$-function (the Euler integral representation is an inversion of this representation)

$$
E_{1}(\tau \mid \omega):=\frac{1}{2 \pi \imath} \int_{-\imath \infty+\epsilon}^{+\imath \infty+\epsilon} d s e^{-s \tau} \Gamma_{1}(s \mid \omega)=\left(\frac{\omega}{2 \pi}\right)^{\frac{1}{2}} e^{-\frac{1}{\omega} \omega^{\omega \tau}}
$$

The standard functional equation on $\Gamma$-function can be written as the following equation on its Fourier transform

$$
\left(\partial_{\tau}+e^{\omega \tau}\right) E_{1}(\tau \mid \omega)=0 .
$$

Recall that $\Gamma$-function allows a representation as the infinite-dimensional integral (1.30), (1.31). The Fourier transform of the integral (1.30) gives a representation of the $U_{1} \times S^{1}$ - equivariant volume of the projectivization $\mathbb{P}\left(\mathcal{M}\left(D_{1}, \mathbb{C}\right)\right)$ of the space of holomorphic maps $\mathcal{M}\left(D_{1}, \mathbb{C}\right)$ of the disk $D_{1}$ into the complex plane. This symplectic space allows Hamiltonian action of $S^{1}$ by rotations of $D_{1}$. The $S^{1}$-fixed points in $\mathbb{P}\left(\mathcal{M}\left(D_{1}, \mathbb{C}\right)\right)$ in homogeneous coordinates (given by coefficients of series expansions $\varphi(z)=\sum_{m=0}^{\infty} \varphi_{m} z^{m}$ of the holomorphic maps $\varphi: D_{1} \rightarrow \mathbb{C}$ ) are enumerated by $n \in \mathbb{Z}_{\geq 0}$ and given by

$$
\varphi_{m}=0, \quad m \neq n .
$$

Local coordinates near $n$-th isolated fixed point are then can be chosen as $\varphi_{m} / \varphi_{n}, m \neq n$. Formal application of the Duistermaat-Heckman formula [DH] gives the following heuristic expression for the Fourier transform

$$
\frac{1}{2 \pi \imath} \int_{-\imath \infty+\epsilon}^{+\imath \infty+\epsilon} d \lambda e^{-\lambda \tau} Z(\lambda, \omega)=\sum_{n=0}^{\infty} \frac{\prod_{m=1}^{\infty}(m \omega)}{\prod_{m=0, m \neq n}^{\infty}((m-n) \omega)} e^{S_{n}}, \quad S_{n}=n \omega \tau,
$$

where we normalize the right hand side by multiplying on $\tau$ - and $n$-independent function $\prod_{m=1}^{\infty}(m \omega)$. The infinite product above is understood via $\zeta$-function regularization. Now formal manipulations give

$$
\frac{1}{2 \pi \imath} \int_{-\imath \infty+\epsilon}^{+\imath \infty+\epsilon} d \lambda e^{-\lambda \tau} Z(\lambda, \omega)=\left(\frac{\omega}{2 \pi}\right)^{\frac{1}{2}} \sum_{n=0}^{\infty} \frac{(-1)^{n}}{n ! \omega^{n}} e^{n \omega \tau}=\left(\frac{\omega}{2 \pi}\right)^{\frac{1}{2}} e^{-\frac{1}{\omega} e^{\omega \tau}}
$$


Thus taking into account the relation $Z(\lambda, \omega)=\Gamma_{1}(\lambda \mid \omega)$ we arrive at (1.45). It is useful to rewrite the series expansion for $E_{1}(\tau \mid \omega)=(\omega / 2 \pi)^{1 / 2} \exp \left(-\frac{1}{\omega} e^{\omega \tau}\right)$ in the following form

$$
E_{1}(\tau \mid \omega)=\left(\frac{\omega}{2 \pi}\right)^{\frac{1}{2}} \sum_{n=0}^{\infty}\left(\frac{\Gamma_{1}(\epsilon-n \omega \mid \omega)}{\Gamma_{1}(\epsilon \mid \omega)} e^{(n \omega-\epsilon) \tau}\right)_{\epsilon \rightarrow+0}
$$

It is easy to verify that the equation (1.46) reduces to the basic functional relation (1.3) on coefficients of the series (1.50).

Now we apply similar approach to higher-dimensional integrals (1.30) to obtain multiple analogs of (1.45) and (1.50). Consider Fourier transformed infinite-dimensional integral (1.30) for $\ell=0$ and arbitrary $r$

$$
E_{r}(\tau \mid \underline{\omega})=\frac{1}{2 \pi \imath} \int_{-\imath \infty+\epsilon}^{+\imath \infty+\epsilon} d \lambda e^{-\lambda \tau} Z(\lambda, \underline{\omega})
$$

where $\underline{\omega}=\left(\omega_{1}, \ldots, \omega_{r}\right)$. We would like to calculate (1.51) explicitly using fixed point localization with respect to the action of the torus $T_{r}$. By (1.31) this gives an integral transform of multiple $\Gamma$-function which can be inverted to obtain multiple analog of the Euler integral representation

$$
\Gamma_{1}(s \mid \omega)=\left(\frac{\omega}{2 \pi}\right)^{\frac{1}{2}} \int_{-\infty}^{+\infty} d \tau e^{s \tau} e^{-\frac{1}{\omega} e^{\omega \tau}} .
$$

Analogously to the case of $r=1$ (see [GLO3]) the set of fixed points of $T_{r}=S^{1} \times \cdots \times S^{1}$ on $\mathbb{P}\left(\mathcal{M}\left(D_{r}, \mathbb{C}\right)\right)$ is enumerated by $r$-tuples $\underline{n}=\left(n_{1}, \ldots, n_{r}\right)$ of non-negative integers. The local coordinates near isolated fixed points are given by $\varphi_{\underline{m}} / \varphi_{\underline{n}}, \underline{m}=\left(m_{1}, \ldots, m_{r}\right) \neq \underline{n}=\left(n_{1}, \ldots, n_{r}\right)$. The sum over fixed points boils down to the following:

$$
E_{r}(\tau \mid \underline{\omega})=\sum_{\underline{n} \in \mathbb{Z}_{\geq 0}^{r}} \frac{\prod_{\underline{m} \in\left(\mathbb{Z}_{\geq 0}^{r}-\{0\}\right)}\langle\underline{m}, \underline{\omega}\rangle}{\prod_{\underline{m} \in\left(\mathbb{Z}_{\geq 0}^{r}-\{\underline{n}\}\right)}\langle\underline{m}-\underline{n}, \underline{\omega}\rangle} e^{S_{\underline{n}}}, \quad S_{\underline{n}}=\langle\underline{n}, \underline{\omega}\rangle \tau .
$$

This formal expression can be rewritten in terms higher $\Gamma$-functions

$$
E_{r}(\tau \mid \underline{\omega})=E_{r}(0 \mid \underline{\omega}) \sum_{\underline{n} \in \mathbb{Z}_{\geq 0}^{r}}\left(\frac{\Gamma_{r}(\epsilon-\langle\underline{n}, \underline{\omega}\rangle \mid \underline{\omega})}{\Gamma_{r}(\epsilon \mid \underline{\omega})} e^{(\langle\underline{n}, \underline{\omega}\rangle-\epsilon) \tau}\right)_{\epsilon \rightarrow+0} .
$$

On the other hand by taking into account (1.31) and (1.51) we obtain the (Fourier transformed) multiple analog of the Euler integral representation for multiple $\Gamma$-functions. Note that it is easy to check that the series (1.54) satisfies the Fourier transformed analog of the basic functional relation (1.9)

$$
\left(\frac{1}{\Gamma_{r-1}\left(\partial_{\tau} \mid \underline{\omega}-\left\{\omega_{a}\right\}\right)}+e^{\omega_{a} \tau}\right) E_{r}(\tau \mid \underline{\omega})=0, \quad a=1, \ldots, r .
$$

Our considerations in this Subsection were mostly heuristic and additional work is obviously needed to make these reasonings precise.

\section{Topological gauge field theories in $d=2$ and $d=4$}

In GLO4 the results of GLO2, GLO3 were generalized to the case of two-dimensional type $A$ topological sigma models with the compact target spaces $\mathbb{P}^{\ell}$. We identify a particular correlation 
function in type $A$ topological sigma model on a disk $D$ with the Whittaker function associated with the maximal parabolic subgroup of $G L_{\ell+1}$. This provides a representation of the Whittaker functions as an infinite-dimensional integral the over space of holomorphic maps of $D$ in $\mathbb{P}^{\ell}$. In explicit calculation of the corresponding correlation function the representation of the topological sigma model with the target space $\mathbb{P}^{\ell}$ via a linear $U(1)$-gauged sigma model with the target space $\mathbb{C}^{\ell+1}$ was used. In GLO4 we also give a mirror dual description of the type $A$ twisted topological $\mathbb{P}^{\ell}$-sigma model in terms of a type $B$ twisted Landau-Ginzburg model reproducing the finite-dimensional integral representation of the Whittaker function GKLO. One shall stress that the correlation functions in type $A$ topological $\mathbb{P}^{\ell}$-sigma model on a disk are closely connected with the counting two-dimensional instantons i.e. holomorphic maps of $\mathbb{P}^{1}$ in $\mathbb{P}^{\ell}$ Gi1, Gi2], GLO1] (the Whittaker functions appear already in instanton counting [Gi3]). However the most direct connection between the Whittaker functions and correlation functions in two-dimensional topological quantum field theories arises when the latter are considered on two-dimensional disks.

To discuss further generalizations of the results of [GLO4] let us note that the calculation of a particular correlation function in topological $\mathbb{P}^{\ell}$-sigma model [GLO4] can be reduced to a calculation of $S^{1} \times U_{\ell+1}$-equivariant symplectic volume of the space of holomorphic maps of the disk $D$ into $\mathbb{P}^{\ell}$. This formulation has obvious relation with the basic setup of the Floer cohomology groups of Lagrangian submanifolds calculated via counting holomorophic disks. There is a well-known four-dimensional analog $[\mathrm{F}]$ (see also e.g. [CJS], $\mathrm{AB}]$ and $[\mathrm{DK}]$ for general facts on instanton moduli spaces) of the theory where the role of the space of holomorphic maps of two-dimensional disks into symplectic manifolds is played by the moduli spaces of instantons i.e. the spaces of gauge equivalence classes of anti self-dual connections on principle $G$-bundles over four-dimensional manifolds with nontrivial boundaries. The cohomology invariants of the instanton moduli spaces (such as e.g. equivariant symplectic volumes) can be conveniently described in terms of topological Yang-Mills theories [W1. Thus one would expect that a generalization of the results of GLO4] to the case of topological four-dimensional Yang-Mills gauge theories would provide an interesting example of higher analog of special function. Let us remark that in four dimensions there is an analog of two-dimensional mirror-symmetry known as $S$-duality. In the case of asymptotically free $\mathcal{N}=2$ Yang-Mills theories with a gauge group $G$ the $S$-dual theory is a $\mathcal{N}=2$ Yang-Mills theory with an abelian gauge group dual to abelian subgroup of the original gauge group $G$ interacting with monopole hypermultiplets. There exists an explicit construction of the mirror duals to twodimensional gauged linear sigma-models $[\mathrm{AV}]$. Thus one might hope that there is a similar effective dual description of the correlation functions in the topological non-abelian Yang-Mills theories via $S$-dual topological theories. Let us stress that the explicit calculations of a particular instanton counting functions in $\mathcal{N}=2$ SUSY Yang-Mills theories were initiated in [LNS] and its relation with Seiberg-Witten geometry was demonstrated in $[\mathrm{N}],[\mathrm{NO}],[\mathrm{NY}]$ by explicit calculations (see also [BE] where the affine Whittaker functions were related with instanton counting in topological Yang-Mills theories). Thus pursuing the analogy with [GLO4 in the four-dimensional case one may hope to find a more direct and conceptional explanation of the relation between instanton counting and Seiberg-Witten solution of $\mathcal{N}=2$ SUSY Yang-Mills theory. In this part of the note we briefly describe basic constructions in topological field theories on two- and four-dimensional non-compact manifolds relevant to the program of deriving dual pairs of integral representations of new special functions associated with topological gauge field theories in four dimensions. We postpone detailed considerations including more general examples for the future publication [GL2]. 


\subsection{Equivariant symplectic volumes of instanton moduli spaces}

Let us first recall basic constructions used in GLO4] with the emphasis on a relation with the Floer cohomology theory (see e.g. [CJS, Gi1]).

Let $X$ be a Kähler manifold with a Kähler form $\omega$. Let $\widetilde{L X}$ be a universal cover of the loop space $L X$ of $X$. Consider a submanifold $\widehat{L X}_{+} \subset \widehat{L X}$ of the loops $\Phi: S^{1} \rightarrow X$ allowing an extension to holomorphic maps of the disk $D, \partial D=S^{1}$ into $X$. This is a sympelctic manifold with the sympelctic structure

$$
\Omega_{2}=\int_{S^{1}} d \sigma \omega_{i \bar{j}}(\varphi, \bar{\varphi}) \delta \varphi^{i} \wedge \delta \bar{\varphi}^{\bar{j}}
$$

where $\Phi$ is locally described by a set of functions $\left(\varphi^{i}(\sigma), \bar{\varphi}^{i}(\sigma)\right)$. The group $S^{1}$ of loop rotations acts on $\left(\widetilde{L X}_{+}, \Omega_{2}\right)$ in a Hamiltonian way and we denote the corresponding momentum by $H_{S^{1}}$. Suppose that $X$ is also supplied with the Hamiltonian action of a compact Lie group $G$ and $\mu(\varphi, \bar{\varphi})$ be the corresponding momentum map $\mu: X \rightarrow \mathfrak{g}^{*}, \mathfrak{g}=\operatorname{Lie}(G)$. The momenta for induced action of $G$ on $\widetilde{L X}_{+}$are then given by

$$
H_{a}=\int_{S^{1}} d \sigma \mu_{a}(\varphi(\sigma), \bar{\varphi}(\sigma)), \quad a=1, \ldots, \operatorname{dim}(G) .
$$

In GLO2, GLO4 the following $S^{1} \times G$-equivariant symplectic volume integrals were considered

$$
Z=\int_{\widetilde{L X}_{+}} e^{\Omega_{2}-\sum_{a} \lambda_{a} H_{a}-\hbar H_{S^{1}}}
$$

where $\Omega_{2}-\sum_{a} \lambda_{a} H_{a}-\hbar H_{S^{1}}$ shall be understood as $S^{1} \times G$-equivariant extension of the symplectic form (2.1). It was demonstrated in GLO2], GLO4 that such integrals for particular $(X, \omega, G)$ provide infinite-dimensional integral representations of special functions such as $\Gamma$-function and various Whittaker functions.

The construction described above allows the following four-dimensional generalization. Let $M$ be a four-dimensional manifold with a boundary $N=\partial M$. One considers the universal cover $\widetilde{\mathcal{A}_{N} / \mathcal{G}}$ of the space $\mathcal{A}_{N} / \mathcal{G}$ of gauge equivalence classes of connections on a principle $G$-bundle over a three-dimensional manifold $N$ (it might be more natural to consider the factorization over subgroup of basic gauge transformations $\mathcal{G}_{0} \subset \mathcal{G}$ i.e. respecting a trivialization at a point in $N$ ). Let $N$ allow an action of an isometry group $G_{N}$ and this action can be extended to $M$. The space $\widetilde{\mathcal{A}_{N} / \mathcal{G}}$ shall be considered as an analog of the space $\widetilde{L X}$ in two-dimensional case and an appropriate subgroup of $G_{N}$ will play the role of the group $S^{1}$ of disk rotations. The analog of the space of holomorphic maps $D \rightarrow X$ is then the space $\mathcal{M}(M, N)$ of gauge equivalence classes of anti self-dual

$G$-connections on $M$ understood as a subspace in $\widetilde{\mathcal{A}_{N} / \mathcal{G}}$ via restriction to the boundary $N$. There is also an action of the group $G$ of global gauge transformations on the moduli space $\mathcal{M}(M, N)$ of anti-self-dual $G$-connections on $M$. In the calculation of the correlation function in $\mathbb{P}^{\ell}$ topological sigma model GLO4 the basic observable in the corresponding linear $U(1)$ gauged sigma model on the disk was the integral $\mathcal{O}=\int_{D} F(A)$ representing the pull back of the standard symplectic form on the target space $\mathbb{P}^{\ell}$. In four-dimensional case the formal analog is given by the integral

$$
\mathcal{O}=\int_{M} \operatorname{Tr} F(A) \wedge F(A)
$$

where $F(A)$ is a curvature of the connection $A$ on a principle $G$-bundle on $M$. 
Let us consider a simple case of $N=S^{1} \times \Sigma, \Sigma$ being a compact two-dimensional surface and $G_{N}=S^{1}$ acts on the first factor by rotations. The symplectic structure on $\mathcal{M}(N, M)$ is given by a restriction of the following two-form

$$
\Omega_{4}=\frac{1}{2} \int_{N=S^{1} \times \Sigma} e_{S^{1}} \wedge \operatorname{Tr} \delta A \wedge \delta A,
$$

where $e_{S^{1}}$ is a lift of constant one-form $d \theta$ on the first factor $S^{1}$ of $N=S^{1} \times \Sigma$.

In analogy with (2.3) we would like to calculate $S^{1} \times G$-equivariant symplectic volume of the space $\mathcal{M}(M, N)$

$$
Z\left(\phi_{0}, \hbar\right)=\int_{\mathcal{M}(M, N)} e^{\Omega_{4}^{\text {equiv }}}, \quad \phi_{0} \in \operatorname{Lie}(G)
$$

where $\Omega_{4}^{\text {equiv }}$ is following $S^{1} \times G$-equivariant extension of the symplectic form (2.5):

$$
\Omega_{4}^{\text {equiv }}=\int_{N=S^{1} \times \Sigma} e_{S^{1}} \wedge \operatorname{Tr}\left(\frac{1}{2} \delta A \wedge \delta A+\phi_{0} F(A)\right)+\hbar S_{C S}(A)
$$

and $S_{C S}(A)$ is the Chern-Simons functional

$$
S_{C S}(A)=\int_{N} \operatorname{Tr}\left(A d A+\frac{2}{3} A^{3}\right) .
$$

This integral basically reduces to the functional integral in the Chern-Simons theory on the boundary $N$ restricted to the connections such that the connections on $N$ can be extended to anti self-dual connections on $M$. Note that the integrand in (2.6) is not invariant with respect to large gauge transformations (related with non-trivial instantons in the bulk). This is consistent with the fact that we consider universal cover $\widetilde{\mathcal{A}_{N} / \mathcal{G}_{0}}$ instead of $\mathcal{A}_{N} / \mathcal{G}_{0}$. In the following Subsection we rewrite this integral using the standard formalizm of topological gauge field theories [W1].

\subsection{Topological field theory representation of symplectic volumes}

Equivariant symplectic volume (2.6) of the moduli spaces of instantons $\mathcal{M}(M, N)$ can be represented as the functional integral in a topological gauge field theories [W1. Let us recall basic construction of the topological gauge theory associated with a principle $G$-bundle $\mathcal{P}_{G}$ over a four-dimensional manifold $M$. Let $\mathfrak{g}=\operatorname{Lie}(G)$ be a Lie algebra of $G$. Topological gauge theory field multiplet $(A, \psi, \phi)$ consists of a connection $A, \mathfrak{g}$-valued odd one form $\psi$ and a $\mathfrak{g}$-valued complex even scalar field $\varphi$. More precisely the last two fields take values in $a d_{\mathfrak{g}}$-bundles associated with $\mathcal{P}_{G}$. The BRST transformations are defined as follows:

$$
\delta A=\psi, \quad \delta \psi=-D \phi, \quad \delta \phi=0,
$$

where $D \phi$ stands for covariant derivative $D_{\mu} \phi=\partial_{\mu} \phi+\left[A_{\mu}, \phi\right]$. Consider additional field multiplet consisting of an anti self-dual two form $\chi$

$$
\chi_{\mu \nu}^{a}=-\chi_{\nu \mu}^{a}=\frac{1}{2} \epsilon_{\mu \nu \rho \tau} \chi^{a \rho \tau},
$$

its BRST partner $H$

$$
\delta \chi=H, \quad \delta H=[\phi, H],
$$


and a pair of even and odd zero forms $\lambda, \eta$

$$
\delta \lambda=\eta, \quad \delta \eta=[\phi, \lambda] .
$$

The action is given by a $\delta$-variation

$$
\begin{gathered}
S=\delta\left(\int_{M} d^{4} x \mathcal{V}\right)=\int_{M} d^{4} x \operatorname{Tr}\left(-2 H^{\mu \nu} F_{\mu \nu}^{+}+\frac{1}{2} \phi D_{\mu} D^{\mu} \lambda-\eta D_{\mu} \psi^{\mu}\right. \\
\left.-\lambda\left[\psi_{\mu}, \psi^{\mu}\right]-\chi^{\mu \nu}\left(D_{\mu} \psi_{\nu}-D_{\nu} \psi_{\mu}-\epsilon_{\mu \nu \rho \tau} D^{\rho} \psi^{\tau}\right)\right),
\end{gathered}
$$

where $\left.\mathcal{V}=\operatorname{Tr}\left(-D_{\mu} \lambda \psi^{\mu}+2 \chi^{\mu \nu} F_{\mu \nu}^{+}\right)\right)$and $F_{\mu \nu}^{+}=\frac{1}{2}\left(F_{\mu \nu}+* F_{\mu \nu}\right)$. After integration over $H$ in the functional integral with the action (2.13) the integral over gauge connections is localized on a subset of anti self-dual connections $F^{+}=0$. Further integration over $\chi$ and $\eta$ leads to the constraints

$$
D_{\mu} \psi_{\nu}-D_{\nu} \psi_{\mu}-\epsilon_{\mu \nu \rho \tau} D^{\rho} \psi^{\tau}=0, \quad D_{\mu} \psi^{\mu}=0 .
$$

These constraints effectively restrict $\psi$ to be a section of the tangent bundle to the moduli space of anit self-dual connections. Thus the functional integral (modulo some subtleties with zero modes [W1] reduces to the integral over odd tangent bundle to the moduli space of instantons.

Now consider an $S^{1} \times G$-equivariant extension of the topological Yang-Mills theory TYM theory on $D \times \Sigma$ where $S^{1}$ acts by rotations of the disk $D$. The $S^{1} \times G$-equivariant BRST operator acts on the topological gauge multiplet as follows (compare with GLO4])

$$
\begin{array}{r}
\delta_{e q} A=\psi, \quad \delta_{e q} \psi=-D \phi+\hbar d\left(\iota_{v} A\right)+\hbar \iota_{v} F(A), \quad \delta_{e q} \phi=0, \\
\delta_{e q} \chi=H, \quad \delta_{e q} H=\hbar \mathcal{L}_{v} \chi, \quad \delta_{e q} \lambda=\eta, \quad \delta_{e q} \eta=\hbar \mathcal{L}_{v} \lambda,
\end{array}
$$

where $v$ is a generator of $S^{1}$. The four-manifold $M=D \times \Sigma$ has the boundary $N=S^{1} \times \Sigma$ and the following observable is $\delta_{e q}$-closed

$$
\mathcal{O}=\int_{S^{1} \times \Sigma} e_{S^{1}} \wedge \operatorname{Tr}\left(\frac{1}{2} \psi \wedge \psi+\phi F(A)\right)+\hbar S_{C S}(A),
$$

where

$$
S_{C S}(A)=\int_{S^{1} \times \Sigma} \operatorname{Tr}\left(A d A+\frac{2}{3} A^{3}\right)
$$

is the Chern-Simons functional. This is precisely the observable we use in (2.6) to define the equivaraint symplectic volume of the moduli space of instantons on non-compact four-manifolds. To calculate the integral (2.6) one can use equivariant localization or/and explicit parametrization of anti self-dual fields via twistor formalizm. Note that one can consider another interesting examples of four-manifolds with non-trivial isometries such as $D \times D$ with a natural action of $S^{1} \times S^{1}$ rotating two disks independently. This leads to a consideration of the Chern-Simons theory on $S^{1} \times D$ i.e. to a potential connection with conformal field theories (compare with [AGT]).

\subsection{On a dual description of equivariant symplectic volumes}

General approach to study topology of instanton moduli spaces via $S$-dual quantum gauge field theories was proposed in [W2] and successfully applied to calculations of the Donaldson invariants of compact four-dimensional manifolds. One would expect that the same approach should work for 
the calculation of equivariant volumes (2.6) on non-compact four-manifolds (see [GW] for related considerations). Let us stress that similar approach based on mirror symmetry successfully works for non-compact two-dimensional surfaces [GLO4]. A mirror dual description of two-dimensional sigma models with the target spaces being compact Kähler manifolds with positive first Chern class in terms of Landau-Ginzburg theories leads to explicit finite-dimensional integral representations of the corresponding equivaraint symplectic volume integrals (2.3).

Let us provide some general remarks on analogy between four-dimensional $S$-duality versus two-dimensional mirror symmetry relevant to calculations of equivariant symplectic volumes. The approach of [W2 can be considered as an application of $S$-duality in the following sense. Recall that $S$-duality transformation of abelain gauge fields relates on-shell gauge field $A$ and its dual $A^{\vee}$ via the constraint $F\left(A^{\vee}\right)=* F(A)$. This relation can be non-trivially generalized to finite non-abelian theories such as $\mathcal{N}=4$ SUSY Yang-Mills theories or finite $\mathcal{N}=2$ SUSY $S U(N)$ YangMills theories with matter multiplets in the fundamental representation by taking into account non-perturbative effects. For asymptotically free theories such as pure $\mathcal{N}=2$ SUSY Yang-Mills theories the $S$-duality relation even more involved. The theory that is dual to microscopic nonabelian $\mathcal{N}=2$ Yang-Mills theory is an abelain theory with monopoles. Heuristically the duality transformation in this case goes as follows. Generically on the moduli space of vacuums the nondiagonal components of the gauge fields are massive and can be "integrated out" in the effective low-energy description. However near points of the moduli space where non-perturbative monopole solutions become massless (and the proper description is in terms of the dual abelain gauge fields) to obtain non-singular description one shall "integrate in" the near-massless monopole field. The resulting theory of dual abelian gauge fields interacting with monopole hypermultiplets can be considered as an $S$-dual description of the original pure $\mathcal{N}=2$ Yang-Mills theory. The case of finite $\mathcal{N}=4$ Yang-Mills theory can be also treated this way by "integrating in" the non-diagonal gauge fields for the dual gauge group.

This description of $S$-duality in four dimensions is completely analogous to the description of the mirror symmetry for $\mathbb{P}^{\ell}$-sigma models realized as $U(1)$-gauged linear sigma models of the fields $\left(X^{i}, \Sigma_{a}\right)$ where $X^{i}, i=1, \ldots,(\ell+1)$ are chiral superfields and $\Sigma$ is a twisted chiral superfield. By integrating out $X^{i}$ one obtains an effective theory of twisted chiral superfield $\Sigma$ with the superpotential of the form

$$
W(\Sigma)=\Sigma \ln \Sigma+\ldots
$$

Now we can "integrate in" additional twisted chiral supermultiplets $Y^{j}$ to obtain the dual effective twisted potential

$$
W(\Sigma, Y)=\Sigma\left(\sum_{j} Y^{j}-r^{2}\right)+\sum_{j=1} e^{Y_{j}}
$$

(see $[\mathrm{AV}]$ for details). This provides a mirror dual Landau-Ginzburg description of $\mathcal{N}=2$ SUSY $\mathbb{P}^{\ell}$ sigma model.

We are interested in calculation of equivaraint symplectic volume of instantons on non-compact four-manifold (2.6). Let us outline the corresponding dual description of the SUSY gauge theories on non-compact manifolds. The Seiberg-Witten solution [SW] of the pure $\mathcal{N}=2$ SUSY $S U(N+1)$ gauge field theory specifies a prepotential $\mathcal{F}(\mathcal{A})$ of the low-energy effective theory depending on $\mathcal{N}=2$ abelian vector superfields $\mathcal{A}^{i}, i=1, \ldots, N+1$. The classical contribution to the prepotential $\mathcal{F}(\mathcal{A})$ is given by $\mathcal{F}_{0}(A)=\frac{1}{2} \tau_{0} \sum_{i=1}^{N} A_{i}^{2}, \tau_{0}=\frac{\theta}{2 \pi}+\imath \frac{4 \pi}{g^{2}}$ and the complete function $\mathcal{F}(A)$ entering the Seiberg-Witten solution encodes a geometry of a family of algebraic curves. The corresponding 
action functional can be written in terms of $\mathcal{N}=1$ chiral and vector superfields $A^{i}$ and $W_{\alpha}^{i}$ as follows:

$$
S=\frac{1}{4 \pi} \operatorname{Im}\left[\int d^{4} x d^{4} \theta \frac{\partial \mathcal{F}(A)}{\partial A^{i}} \bar{A}^{i}+\frac{1}{2} \int d^{4} x d^{2} \theta \frac{\partial^{2} \mathcal{F}(A)}{\partial A^{i} \partial A^{j}} W_{\alpha}^{i} W_{\alpha}^{j}\right] .
$$

Note that the action (2.20) the $\mathcal{N}=2$ vector multiplet with a general prepotential $\mathcal{F}(\mathcal{A})$ can be considered as an integral of a four-observable $\mathcal{O}^{(4)}$ constructed from a zero-observable $\mathcal{O}^{(0)}=\mathcal{F}(\phi)$ by the standard descent procedure $d \mathcal{O}^{(n)}=\delta \mathcal{O}^{(n+1)}$. Similar to considerations in GLO3]. On the non-compact four-manifold there is a non-trivial boundary contribution breaking $\mathcal{N}=2$ SUSY invariance of the theory (this is an analog of the Warner problem in two-dimensional SUSY theories)

$$
\delta S=\int_{M} d \mathcal{O}^{(3)}=\int_{N=\partial M}\left(\frac{\partial^{2} \mathcal{F}}{\partial \phi^{i} \partial \phi^{j}} F^{i} \psi^{j}+\frac{1}{3 !} \frac{\partial^{3} \mathcal{F}}{\partial \phi^{i} \partial \phi^{j} \partial \phi^{k}} \psi^{i} \psi^{j} \psi^{k}\right),
$$

where $\phi^{i}$ and $\psi^{i}$ are components of the abelian $\mathcal{N}=2$ supermultiplet $\mathcal{A}^{i}$ consisting of abelain vector field $A_{\mu}^{i}$, two Weyl fermions $\lambda^{i}, \psi^{i}$ and a complex scalar field $\phi^{i}$. We also denote $F^{i}$ the curvature of the gauge field $A^{i}$. Consider now the case of $M=D \times \Sigma, N=\partial M=S^{1} \times \Sigma$ with an action of $S^{1}$ by rotations of the disk $D$. In $S^{1}$-equivariant case, similarly to the two-dimensional case GLO3, GLO4, this boundary contribution can be canceled by the variation of a boundary term expressed through the Seiberg-Witten prepotential $\mathcal{F}(\phi)$. In two dimensions this boundary term enters the expression of the integrand of the Givental type finite dimensional integral representation of the correlation function in topological sigma models. In the case of gauge fields in four-dimensional one expects that the Seiberg-Witten prepotential provides an effective description of the integrand of the corresponding integral representation of the equivariant symplectic volume (2.6). It is reasonable to guess that the precise description of the integrand in the four-dimensional analog of the MellinBarnes integral representation should be given in terms of the dual theory of monopoles interacting with the dual abelian gauge fields.

\subsection{Integral representations of vortex counting functions}

In type $B$ topological Landau-Ginzburg sigma-models correlation functions are naturally given by periods of holomorphic differential forms. Thus for instance the dual type $B$ description of the type $A$ topological sigma model on a disk with the target space $\mathbb{P}^{\ell}$ leads to a finite-dimensional integral representation of the corresponding Whittaker function [GLO4]. The integrand of this integral representation is directly related with the superpotential of the dual Landau-Ginzburg theory. Note that the arising Whittaker function is closely related to the instanton counting functions in the corresponding $\mathbb{P}^{\ell}$ two-dimensional sigma model [Gi1] (see also [GLO1]).

Taking into account the analogy between counting instantons in two and four dimensions one might expect that the instanton counting function of [LNS] can be also recasted in the compact integral form to provide a direct relation with the Seiberg-Witten prepotential as it was outlined at the end of the previous Subsection (for a direct comparison of the asymptotic of the instanton counting function with Seiberg-Witten solution see [N], [NO]). We postpone the construction of this integral representation to another occasion while in the rest of this note we consider a degenerate version of the instanton counting function responsible for counting of two-dimensional vortexes (see e.g. [JT] for discussion of vortexes). The main result of this section is the compact Mellin-Barnes type integral representation (2.35) of the vortex counting function (2.27).

Recall that the instanton counting function (up to the classical and one loop contributions) in 
$\mathcal{N}=2$ SUSY $S U(N)$-gauge theory interacts with $N_{f}$ hypermultiplets in the fundamental representation can be written in the form of the infinite series [LNS]

$$
\mathcal{Z}^{i n s t}(a, \tau, \omega, m)=1+\sum_{k=1}^{\infty} e^{2 \pi \imath \tau k} \mathcal{Z}_{k}^{\text {inst }}(a, m, \omega),
$$

where

$$
\begin{gathered}
\mathcal{Z}_{k}^{\text {inst }}(a, \omega, m)=\frac{1}{k !} \frac{\left(\omega_{1}+\omega_{2}\right)^{k}}{\left(\omega_{1} \omega_{2}\right)^{k}} \int \prod_{j=1}^{k} \frac{d \phi^{j}}{2 \pi \imath} \prod_{i<j} \frac{\left(\phi_{i}-\phi_{j}\right)^{2}\left(\left(\phi_{i}-\phi_{j}\right)^{2}-\left(\omega_{1}+\omega_{2}\right)^{2}\right.}{\left(\left(\phi_{i}-\phi_{j}\right)^{2}-\omega_{1}^{2}\right)\left(\left(\phi_{i}-\phi_{j}\right)^{2}-\omega_{2}^{2}\right)} \times \\
\times \prod_{j=1}^{k} \frac{\prod_{\alpha=1}^{N_{f}}\left(\phi_{j}+m_{\alpha}\right)}{\prod_{l=1}^{N}\left(\phi_{j}-a_{l}\right)\left(\phi_{j}-a_{l}+\omega_{1}+\omega_{2}\right)} .
\end{gathered}
$$

The vortex counting function (see e.g. [Shad]) can be defined by taking a limit of the instanton counting function

$$
\mathcal{Z}^{\text {vortex }}\left(a, \tau, m, \omega_{1}\right)=\lim _{\omega_{2} \rightarrow \infty} \mathcal{Z}^{\text {inst }}\left(a, \tau+\frac{N}{2 \pi \imath} \ln \omega_{2}, m, \omega_{1}, \omega_{2}\right)
$$

The limit can be taken explicitly to obtain (we use simplify notations $\omega:=\omega_{1}$ below)

$$
\mathcal{Z}^{\text {vortex }}(a, \tau, \omega, m)=1+\sum_{k=1}^{\infty} e^{2 \pi \imath \tau k} \mathcal{Z}_{k}(a, m, \omega),
$$

where

$$
\mathcal{Z}_{k}(a, \omega, m)=\frac{1}{k !} \frac{1}{\omega^{k}} \int \prod_{j=1}^{k} \frac{d \phi^{j}}{2 \pi \imath} \prod_{i<j}^{k} \frac{\left(\phi_{i}-\phi_{j}\right)^{2}}{\left(\phi_{i}-\phi_{j}\right)^{2}-\omega^{2}} \prod_{j=1}^{k} \frac{\prod_{\alpha=1}^{N_{f}}\left(\phi_{i}+m_{\alpha}\right)}{\prod_{l=1}^{N}\left(\phi_{j}-a_{l}\right)}
$$

Here the integration goes over $\mathbb{R}^{k}$ and we imply that $a_{l}$ and $\omega$ have small positive imaginary parts. Let us multiply (2.25) by a perturbative contribution $\mathcal{Z}^{\text {pert }}$

$$
\begin{gathered}
\mathcal{Z}(a, \tau, m, \omega)=\mathcal{Z}^{\text {pert }}(a, \tau, m, \omega) \mathcal{Z}^{\text {vortex }}(a, \tau, m, \omega), \\
\mathcal{Z}^{\text {pert }}(a, \tau, m, \omega)=\frac{\prod_{\alpha=1}^{N_{f}} \prod_{p=1}^{N} \Gamma_{1}\left(a_{p}+m_{\alpha}+\omega \mid \omega\right)}{\prod_{p \neq q}^{N} \Gamma_{1}\left(a_{p}-a_{q} \mid \omega\right)} .
\end{gathered}
$$

The integral (2.26) over $k$-vortex moduli space can be expressed as a sum over residues (see [N], [NO] for similar calculations in the case of instantons)

$$
\mathcal{Z}_{k}(a, \omega)=\sum_{|\underline{k}|=k} \frac{1}{\underline{k} ! \omega^{k}} \frac{\prod_{f=1}^{N_{f}} \prod_{p=1}^{N} \prod_{i_{p}=1}^{k_{p}}\left(a_{p}+m_{f}+\left(i_{p}-1\right) \omega\right)}{\prod_{l \neq m}^{N} \prod_{i_{l}=1}^{k_{l}}\left(a_{l}-a_{m}+\left(k_{l}-k_{m}-i_{l}\right) \omega\right)},
$$

where the sum goes over partitions $\underline{k}=\left(k_{1}, k_{2}, \ldots, k_{N}\right), k_{l} \in \mathbb{Z}_{\geq 0},|\underline{k}|=k_{1}+k_{2}+\ldots+k_{N}$. Taking into account the following simple identities

$$
\frac{\Gamma(x+n+1)}{\Gamma(x)}=\prod_{p=0}^{n}(x+p), \quad \frac{\Gamma(x)}{\Gamma(x-n)}=\prod_{p=1}^{n}(x-p), \quad n>0,
$$




$$
\left.\frac{\partial}{\partial x}\left(\frac{1}{\Gamma(x-k)}\right)\right|_{x=0}=(-1)^{k} k !
$$

we have

$$
\begin{gathered}
\frac{1}{\prod_{l \neq m}^{N} \prod_{i_{l}=1}^{k_{l}}\left(a_{l}+\left(k_{l}-i_{l}\right) \omega-\left(a_{m}+k_{m} \omega\right)\right)}=\frac{\prod_{l \neq m}^{N} \Gamma_{1}\left(a_{l}-\left(a_{m}+k_{m} \omega\right) \mid \omega\right)}{\prod_{l \neq m}^{N} \Gamma_{1}\left(a_{l}+k_{l} \omega-\left(a_{m}+k_{m} \omega\right) \mid \omega\right)}, \\
\prod_{f=1}^{N_{f}} \prod_{p=1}^{N} \prod_{i_{p}=1}^{k_{p}}\left(a_{p}+m_{f}+\left(i_{p}-1\right) \omega\right)
\end{gathered}
$$

Thus the vortex generating function can be rewritten as follows:

$$
\begin{gathered}
\mathcal{Z}^{\text {vortex }}(a, \tau, m, \omega)=\frac{1}{\prod_{f=1}^{N_{f}} \prod_{p=1}^{N} \Gamma_{1}\left(a_{p}+m_{f}+\omega \mid \omega\right)} \sum_{k=0}^{\infty} e^{2 \pi \imath \tau k} \sum_{|\underline{k}|=k} \frac{1}{\underline{k} ! \omega^{k}} \\
\times \prod_{f=1}^{N_{f}} \prod_{p=1}^{N} \Gamma_{1}\left(a_{p}+k_{p} \omega+m_{f} \mid \omega\right) \prod_{l \neq m}^{N} \frac{\Gamma_{1}\left(a_{l}-\left(a_{m}+k_{m} \omega\right) \mid \omega\right)}{\Gamma_{1}\left(a_{l}+k_{l} \omega-\left(a_{m}+k_{m} \omega\right) \mid \omega\right)} .
\end{gathered}
$$

Let us consider the following multiple integral

$$
\begin{gathered}
\widetilde{\mathcal{Z}}(a, \tau, m, \omega)=\int_{\mathcal{S}} \prod_{j=1}^{N} \frac{d \phi_{j}}{2 \pi \imath} e^{2 \pi \imath\left(\tau+\frac{1}{2}\right) \sum_{j=1}^{N}\left(\phi_{j}-a_{j}\right) / \omega} \frac{\prod_{j=1}^{N} \prod_{l=1}^{N} \Gamma_{1}\left(a_{l}-\phi_{j} \mid \omega\right)}{\prod_{i \neq j} \Gamma_{1}\left(\phi_{i}-\phi_{j} \mid \omega\right)} \times \\
\times \frac{\prod_{j=1}^{N} \prod_{\alpha=1}^{N_{f}} \Gamma_{1}\left(\phi_{j}+m_{\alpha} \mid \omega\right)}{\prod_{j \neq i} \Gamma_{1}\left(a_{j}-a_{i} \mid \omega\right)},
\end{gathered}
$$

where the integration contour $\mathcal{S}$ encloses only the poles of $\Gamma_{1}\left(a_{l}-\phi_{j} \mid \omega\right)$ (i.e we take $a_{l} \rightarrow a_{l}+\imath 0$ and $\left.m_{f} \rightarrow m_{f}+\imath 0\right)$. The integral is given by as sum over residues at $\phi_{j}=a_{l}+k_{l} \omega$ such that different $\phi_{j}$ correspond to different $a_{l}$. Taking into account that the integrand is symmetric with respect to interchange of $\phi_{j}$ we can take, up to a simple symmetric factor, $\phi_{j}=a_{j}+k_{j} \omega$. Then the evaluation of the residues leads to identification of the integral expression $\widetilde{\mathcal{Z}}(a, \tau, m, \omega)$ given by (2.35) with the vortex counting function (2.27), (2.28), (2.34).

\section{References}

[AV] M. Aganagic, C. Vafa, Perturbative Derivation of Mirror Symmetry, [arXiv:hep-th/0209138].

[AGT] L.F. Alday, D. Gaiotto, and Y. Tachikawa, Liouville Correlation Functions from Four-dimensional Gauge Theories, Lett. Math. Phys. 91 (2010), 167-197; [arXiv:0906.3219].

[AB] D.M. Austin, P.J. Braam, Morse-Bott theory and equivariant cohomology, The Floer Memorial Volume, Birkhäuser Verlag, Progr. Math. 133 (1995), 123-183.

[Ba] E.W. Barnes, On the theory of the multiple gamma functions, Trans. Cambridge Philos. Soc. 19 (1904), $374-425$. 
[BE] A. Braverman, P. Etingof, Instanton counting via affine Lie algebras. II: From Whittaker vectors to the Seiberg-Witten prepotential, [arXiv:math/0409441] .

[B] D. Bump, Automorphic Forms and Representations, Cambridge Univ. Press, Cambridge, 1998.

[CJS] R.L. Cohen, J.D.S. Jones, G.B. Segal, Floers infinite dimensional Morse theory and homotopy theory, The Floer Memorial Volume, Birkhäuser Verlag, Progr. in Math. 133 (1995), 287-325.

[DO] F.A. Dolan, H. Osborn, Applications of the Superconformal Index for Protected Operators and q-Hypergeometric Identities to $N=1$ Dual Theories, Nucl. Phys. B818 (2009), 137-178; [arXiv:0801.4947].

[DK] S.K. Donaldson, P.B. Kronheimer, The Geometry of Four-Manifolds, Clarendon Press, Oxford, 1990.

[DH] J. J. Duistermaat, G. J. Heckman, On the variation in the cohomology of the symplectic form of the reduced phase space, Invent. Math. 69 no. 2 (1982), 259-268.

[F] A. Floer, An instanton invariants for 3-manifolds, Commun. Math. Phys., 118 (1988), 215-240.

[GW] D. Gaiotto, E. Witten, Supersymmetric Boundary Conditions in $\mathcal{N}=4$ Super Yang-Mills Theory, [arXiv:0804.2902].

[GKL] A. Gerasimov, S. Kharchev, D. Lebedev, Representation theory and quantum inverse scattering method: the open Toda chain and the hyperbolic Sutherland model, Int. Math. Res. Notices, 17 (2004), 823-854.

[GKLO] A. Gerasimov, S. Kharchev, D. Lebedev, S. Oblezin, On a Gauss-Givental representation of quantum Toda chain wave function, Int. Math. Res. Notices, (2006), 1-23; [arXiv:math.RT/0505310] .

[GLO1] A. Gerasimov, D. Lebedev, S. Oblezin, On q-deformed $\mathfrak{g l}_{\ell+1}$-Whittaker functions I, II,III, Commun. Math. Phys. 294 (2010), 97-119; [arXiv:0803.0145] ; Commun. Math. Phys. 294 (2010), 121-143; [arXiv:0803.0970]; Lett. Math.Phys. 97 (2011), 1-24; [arXiv:0805.3754].

[GLO2] A. Gerasimov, D. Lebedev, S. Oblezin, Archimedean L-factors and Topological Field Theories I, Commun. in Number Theory and Physics, 5 (1) 2011; [arXiv:0906.1065].

[GLO3] A. Gerasimov, D. Lebedev, S. Oblezin, Archimedean L-factors and Topological Field Theories II, Commun. in Number Theory and Physics, 5 (1) 2011; [arXiv:0909.2016].

[GLO4] A. Gerasimov, D. Lebedev, S. Oblezin, Parabolic Whittaker Functions and Topological Field Theories I, Commun. in Number Theory and Physics, 5 (1) 2011; [arXiv:1002.2622].

[GLO5] A. Gerasimov, D. Lebedev and S. Oblezin, From Archimedean L-factors to Topological Field Theories, Lett. Math. Phys. 96 (2011), 285-297; Mathematische Arbeitstagung, June 2009, MPIM 2009-401.

[G] A. Gerasimov, A Quantum Field Theory Model of Archimedean Geometry, talk at Rencontres Itzykson 2010: New trends in quantum integrability, 21-23 June, 2010, IPhT Saclay, France (see link to slides on the webpage of the conference).

[GL1] A.A. Gerasimov, D.R. Lebedev, Representation Theory over Tropical Semifield and Langlands Correspondence, [arXiv:1011.2462].

[GL2] A.A. Gerasimov, D.R. Lebedev, to appear.

[GS1] A.A. Gerasimov, S.L. Shatashvili, Higgs Bundles, Gauge Theories and Quantum Groups, Commun. Math. Phys. 277 (2008) 323; [arXiv:hep-th/0609024] 
[GS2] A.A. Gerasimov, S.L. Shatashvili, Two-Dimensional Gauge Theories and Quantum Integrable Systems, in From Hodge Theory to Integrability and TQFT: tt*-geometry, pp. 239-262, R. Donagi and K. Wendland, Eds., Proc. of Symposia in Pure Mathematics Vol. 78, American Mathematical Society Providence, Rhode Island; [arXiv:0711.1472].

[Gi1] A. Givental, Homological geometry I. Projective hypersurfaces, Selecta Mathematica, New Series Volume 1, 2 (1995), 325-345.

[Gi2] A. Givental, Equivariant Gromov - Witten Invariants, Int. Math. Res. Notices 1996, 13, 613-663; [arXiv:alg-geom/9603021].

[Gi3] A. Givental, Stationary Phase Integrals, Quantum Toda Lattices, Flag Manifolds and the Mirror Conjecture. Topics in Singularity Theory, Amer. Math. Soc. Transl. Ser., 2 180, AMS, Providence, Rhode Island, 1997, 103-115 [arXiv:alg-geom/9612001] .

[JT] A. Jaffe, C. Taubes, Vortices and Monopoles, Progress in Physics 2, Birkhäuser, 1980.

[KL] S. Kharchev, D. Lebedev, Eigenfunctions of $G L(N, R)$ Toda chain: The Mellin-Barnes representation, JETP Lett. 71 (2000), 235-238.

[KMMR] J. Kinney, J.M. Maldacena, S. Minwalla, S. Raju, An Index for 4 dimensional Super Conformal Theories, Commun. Math. Phys. 275 (2007), 209, [arXiv:hep-th/0510251].

[K] M. Kontsevich, Enumeration of rational curves via torus action, [arXiv:hep-th/9405035].

[Ku1] N. Kurokawa, Multiple sine functions and Selberg zeta functions, Proc. Japan Acad., 67A (1991), 61-64 .

[Ku2] N. Kurokawa, Multiple zeta functions: an example, Adv. Stud. Pure Math., 21 (1992), 219-226.

[L] An introduction to the Langlands program, Lectures presented at the Hebrew University of Jerusalem, Jerusalem, March 12-16, 2001. Edited by J. Bernstein and S. Gelbart. Birkhäuser Boston, Inc., Boston, MA, 2003.

[LNS] A. Losev, N. Nekrasov, S.L. Shatashvili, Testing Seiberg-Witten Solution, NATO Advanced Study Institute on Strings, Branes and Dualities (Cargese, 1997), [arXiv:hep-th/9801061].

[Ma] Yu.I. Manin, Lectures on zeta functions and motives (according to Deninger and Kurokawa), In: Columbia University Number Theory Seminar, Astérisque, 228 (1995), 121-164.

[MNS] G.W. Moore, N. Nekrasov, S. Shatashvili, Integrating over Higgs Branches, Commun. Math. Phys. 209 (2000), 97; [arXiv:hep-th/9712241].

[NY] H. Nakajima, K. Yoshioka, Lectures on instanton counting, [arXiv:math/0311058].

[N] N. A. Nekrasov, Seiberg-Witten Prepotential From Instanton Counting, Adv. Theor. Math. Phys. 7 (2004), 831-864; [arXiv:hep-th/0206161v1] .

[NO] N. Nekrasov, A. Okounkov, Seiberg-Witten Theory and Random Partitions, In: The Unity of Mathematics in honor of the 90th birthday of I.M. Gelfand, Progress in Mathematics 244, Birkhäuser (2005), [arXiv:hep-th/0306238].

[NS] N.A. Nekrasov, S.L. Shatashvili, Quantization of Integrable Systems and Four Dimensional Gauge Theories, arXiv:0908.4052].

[NW] N. Nekrasov, E. Witten, The Omega Deformation, Branes, Integrability, and Liouville Theory, [arXiv: 1002.0888].

[SW] N. Seiberg, E. Witten, Electric-Magnetic Duality, Monopole Condensation, And Confinement in $N=$ 2 Supersymmetric Yang-Mills Theory, Nucl. Phys. B426 (1994), 19-52. 
[R1] C. Römelsberger, Counting chiral primaries in $N=1 d=4$ superconformal field theories, Nucl. Phys. B747 (2006) 329-353; [arXiv:0510060].

[R2] C. Römelsberger, Calculating the Superconformal Index and Seiberg Duality, [arXiv:0707.3702] .

[Shad] S. Shadchin, On F-term contribution to effective action, JHEP $\mathbf{0 8}$ (2007), 052; [arXiv:hep-th/0611278].

[S] V. P. Spiridonov, Russian Math. Surveys 63 (3) (2008), 405; [arXiv:0805.3135] ; V.P. Spiridonov, G.S. Vartanov, Elliptic hypergeometry of supersymmetric dualities, [arXiv:0910.5944].

[W1] E. Witten, Topological Quantum Field Theory, Commun. Math. Phys. 117 (1988), 353.

[W2] E. Witten, Monopoles and Four-Manifolds, Math. Res. Lett. 1 (1994), 769-796; [arXiv:hep-th/9411102].

[W3] E. Witten, A New Look At The Path Integral Of Quantum Mechanics, [arXiv:1009.6032].

A.G. Institute for Theoretical and Experimental Physics, 117259, Moscow, Russia; School of Mathematics, Trinity College Dublin, Dublin 2, Ireland;

Hamilton Mathematics Institute, Trinity College Dublin, Dublin 2, Ireland;

D.L. Institute for Theoretical and Experimental Physics, 117259, Moscow, Russia; 